Journal of Mathematics and Informatics

Vol. 7, 2017, 63-71

ISSN: 2349-0632 (P), 2349-0640 (online)

Published 20 April 2017

www.researchmathsci.org

DOI: http://dx.doi.org/10.22457/jmi.v7a8

Journal of

Mathematics and

Informatics

\title{
A Model of Based on Z-number and Fuzzy Analytic Hierarchy Process
}

\author{
Sheng-sheng Zhang \\ School of Science, Chongqing University of Posts and Telecommunications \\ Chongqing - 400065, Chongqing, China. \\ E-mail: shengsheng_zhang@yeah.net
}

Received 8 April 2017; accepted 13 April 2017

\begin{abstract}
Although the Fuzzy Analytic Hierarchy Process is one Multi-Criteria Decision Making techniques, it does not consider the reliability of information. The Z-number contains both uncertain variable and its reliability, which has been applied to uncertain environment including decision making, risk assessment, linear programming etc. In this paper, a new method of Multi Criteria Decision Making based on Z-number and Fuzzy Analytic Hierarchy Process is proposed, where the evaluation of each alternative with respect to each criterion is described as a Z-number, including both the evaluation and its reliability from evaluators. Finally, a practical example illustrates the steps and effectiveness of the method.
\end{abstract}

Keywords: Fuzzy Analytic Hierarchy Process; Multi Criteria Decision Making; Uncertainty; Reliability; Z-number

AMS Mathematics Subject Classification (2010): 03E72, 90C48

\section{Introduction}

In the real world, risk assessment is ubiquitous. However, due to the uncertainty of information, to the risk assessment has brought great challenges. Therefore, how to correctly assess the risk level has become a hot topic for everyone to study. There are many models and tools to solve this problem, such as probability, game theory, utility function and so on. In 1970, Zadeh and Bellman [1] first proposed the fuzzy decision making model. From then on, many researchers have carried out an in-depth study on Fuzzy Multi Criteria Decision Making (FMCDM) [2]. Most studies show that when uncertainty is not probabilistic, it is rather imprecise or even vague. In some cases, uncertainty arises because of the vagueness of meaning or linguistic terms in people's natural language, fuzzy linguistic approach [3], etc.

Fuzzy Analytic Hierarchy Process (FAHP) [4] is a comprehensive evaluation method combining fuzzy comprehensive evaluation and Analytic Hierarchy Process (AHP) [5], which has wide application in system evaluation, efficiency evaluation and system optimization. It is a combination of qualitative and quantitative evaluation model, generally first determine the set of factors using the chromatographic analysis, and then use fuzzy comprehensive evaluation to determine the results. Fuzzy method is in the 


\section{Sheng-sheng Zhang}

hierarchy method, if the two are integrated with each other, will improve the reliability of evaluation results. For example, in 2010, Khademi Hamidi [6] used FAHP as an effective decision-making method, which could consider different criteria in the pre-known basic relevant conditions to make the appropriate selection. In 2015, Mardani et al [7] summed up the combination methods of fuzzy set [8] and MCDM systematically, as well as the applications and methods of the FMCDM techniques.

In 2011, Zadeh proposed the Z-number theory [9], which relates to the issue of reliability of information. It is an ordered pair of fuzzy numbers and has two components, $\mathrm{Z}=(\mathrm{A}, \mathrm{B})$. The first component, $\mathrm{A}$, is a restriction on the values which a real-valued uncertain variable, $\mathrm{X}$, is allowed to take. The second component, $\mathrm{B}$, is a measure of reliability of the first component. And A and B are described in a natural language. It is a new concept which has more power to describe the knowledge of human being and will be widely used in the decision analysis. However, it is not treated as general numbers because which is much simpler to compute with general numbers than with Z-numbers. Fundamentally, Z-number is a step that formalizes the ability of people to make rational decisions in an environment of uncertainty. Compared with the traditional fuzzy number [10], Z number has a stronger ability to describe the knowledge of human. Recently, Bingyi et al. [11] proposed a method to solve the problem of FMCDM by using Znumber. In order to convert Z-numbers to explicit numbers for the calculation of the decision making, the method requires that the two components of Z-numbers should be the triangular fuzzy numbers [12].

In this paper, we propose a method of FMCDM based on FAHP and Z-number (Z-FAHP). Finally, we illustrate an example to clarify the steps and effectiveness of the Z-FAHP. And we use the data from Nezarat et al. [13].

This article is organized as follows. The second part introduces some basic concepts and definitions that will be used following section. In third part, we propose the Z-FAHP and give its procedure in detail. In fourth part, we give an example of using the Z- FAHP. Lastly, we present conclusions and future direction.

\section{Preliminaries}

\subsection{Fuzzy set, fuzzy numbers and Z-number}

Definition 2.1.1. A fuzzy set [14] $A$ is defined on a $X$ may be given as:

$$
A=\left\{\left(x, \mu_{A}(x)\right) \mid \mu_{A}(x) \in[0,1], x \in X\right\}
$$

where $\mu_{A}(x)$ is a continuous mapping from $R$ to the closed interval $[0,1]$. It is the membership function of $A$. The value of $\mu_{A}(x)$ describes the degree of belongingness of $x \in X$ in $A$ [15].

Definition 2.1.2. The Triangular Fuzzy Numbers (TFN) [12] of $A(A-T F N)$ is one of the many membership functions of $A . A-T F N$ is defined as $(a, b, c)$. The parameter $a$ gives the maximal grade of $\mu_{A}(x)$. The parameter $b$ and $c$ are the lower and upper bounds which limit the field of the possible evaluation [16]. The TFN has linear representations on its left and right side such that its membership function can be defined as: 
A Model of Based on Z-number and Fuzzy Analytic Hierarchy Process

$$
\mu_{A}(x)=\left\{\begin{array}{cl}
(x-a) /(b-a) & a \leq x \leq b \\
(c-x) /(c-b) & b \leq x \leq c, \text { where } a \leq b \leq c \\
0 & \text { otherwise }
\end{array}\right.
$$

The $A-T F N$ is shown in Figure 1 .

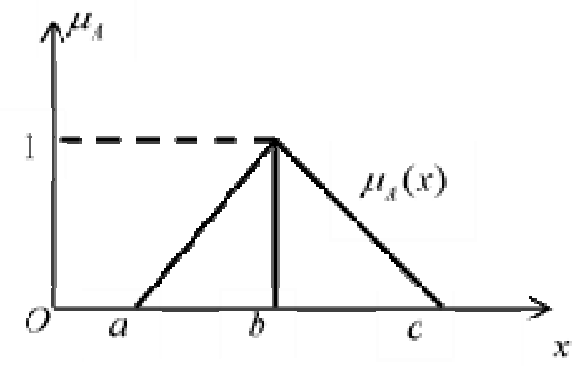

Figure 1: Triangular fuzzy numbers

There are a number of operations on TFN, which are described in detail [12]. According to the needs of the model, the article deals with following three operations. To this end, we define two TFN $A$ and $B$ by the triplets $A=\left(a_{1}, b_{1}, c_{1}\right)$ and $B=\left(a_{2}, b_{2}, c_{2}\right)$. Details as follow:

Addition:

$$
A+B=\left(a_{1}+a_{2}, b_{1}+b_{2}, c_{1}+c_{2}\right)
$$

Multiplication:

$$
A \cdot B=\left(a_{1} \cdot a_{2}, b_{1} \cdot b_{2}, c_{1} \cdot c_{2}\right)
$$

Inverse:

$$
\left(a_{1}, b_{1}, c_{1}\right)^{-1} \approx\left(1 / c_{1}, 1 / b_{1}, 1 / a_{1}\right)
$$

Here $\approx$ is approximately equal to.

Definition 2.1.3. A Z-number [9] is an ordered pair of fuzzy numbers, $Z=(A, B)$. It is associated with a real-valued uncertain in variable, $X$, with the first component, $A$, playing the role of a fuzzy number restriction, $R(x)$, on the values which $X$ is $A$, where $A$ is a fuzzy set. The second component, $B$, is referred to as a measure of reliability for the first component.

\subsection{Fuzzy analytic hierarchy process (FAHP) [17]}

The conventional AHP method is incapable of handling the uncertainty and vagueness involved in the mapping of one's preference to an exact number or ratio. The major difficulty with classical AHP is its inability in mapping human judgments. It uses both qualitative and quantitative variables.

Although the AHP is to capture the expert's knowledge, the traditional AHP is still cannot reflect the human thinking style. In the comparison of alternatives, it uses an accurate value to express the thought of decision maker, and the results of AHP are subjectively influenced by experts and so on. To solve these problems, FAHP was 


\section{Sheng-sheng Zhang}

developed. FAHP provides decision makers with interval judgements rather than a fixed value.

\subsection{Converting $Z$-numbers to crisp numbers}

To convert a $Z$-number on outcomes and probabilities. We can assume a Z-number, $\mathrm{Z}=(A, B)$ where

$$
A=\left\{\left(x, \mu_{A}(x)\right) \mid \mu_{A}(x) \in[0,1], x \in X\right\}
$$

and

$$
B=\left\{\left(x, \mu_{B}(x)\right) \mid \mu_{B}(x) \in[0,1], x \in X\right\} .
$$

Here $\mu_{A}=\left(a_{1}, b_{1}, c_{1}\right)$ and $\mu_{B}=\left(a_{2}, b_{2}, c_{2}\right)$ are TFN. More details see [11].

1) Converting the describing reliability $(B)$ of $A$ into a crisp number.

In order to convert $B$ into a crisp number, we use the method proposed by Ali Azadeh et al [18].

$$
\alpha=\frac{\int x \mu_{B}(x) d x}{\int \mu_{B}(x) d x}
$$

where $\int$ denotes an algebraic integration. Now we can describe Z-number as

$$
\mathrm{Z}=(A, \alpha)
$$

2) Convert weighted $Z$-number to regular fuzzy number

Add the weight of the $B$ to the $A$. Weighted Z-number can be denoted as

$$
\tilde{Z}=\left\{\left(x, \mu_{\tilde{A}}(x)\right) \mid \mu_{\tilde{A}}(x)=\sqrt{\alpha} \mu_{A}, x \in X\right\}
$$

where $\tilde{Z}$ is also denoted

$$
\tilde{Z}=(\sqrt{\alpha} a, \sqrt{\alpha} b, \sqrt{\alpha} c)
$$

So far, a Z-number has been converted to a crisp number.

\section{Z- FAHP model}

Although FAHP provides a good reference value for decision-makers, it still has ambiguity in language description and can't respond well to the evaluators' natural language, fuzzy linguistic approach and so on. Z-number appears to be a good solution to this defect. Therefore, we choose the advantages of FAHP and Z-number respectively. Combining them together to form a new method, which is also called Z- FAHP. Specific steps are as follows:

1) Construction of the detailed hierarchy of the problem

The hierarchy is constructed taking all the criteria, sub-criteria and alternatives specific to the research problem. The hierarchy is structured from the top (performance evaluation of technical institutions) through the intermediate levels (main and sub-criteria on which subsequent levels depend) to the bottom level (the list of technical institutions).

2) Constructing a pair wise comparison matrix

Once the hierarchy was established and a series of questions were asked to direct pair wise comparisons, each expert performed a pair wise comparison. Assuming expert gives his or her opinion as follows:

$$
A=\left(a_{1}, b_{1}, c_{1}\right) \quad B=\left(a_{2}, b_{2}, c_{2}\right)
$$


A Model of Based on Z-number and Fuzzy Analytic Hierarchy Process

where $A$ and $B$ are TFN.

The expert's opinion can be described to a Z-number as:

$$
\mathrm{Z}=(A, B)=\left[\left(a_{1}, b_{1}, c_{1}\right),\left(a_{2}, b_{2}, c_{2}\right)\right]
$$

Firstly, according to the Eq. 6, we can convert $B$ to a crisp number. Secondly, we add the weight of $B$ to the $A$ according Eq. 7 .

$$
\mathrm{Z}=(A, \alpha)=\left[\left(a_{1}, b_{1}, c_{1}\right), \alpha\right]
$$

Thirdly, convert $\mathrm{Z}=(A, \alpha)$ to a crisp number according Eq. 8 and Eq. 9.

$$
\tilde{Z}=\left(\sqrt{\alpha} a_{1}, \sqrt{\alpha} b_{1}, \sqrt{\alpha} c_{1}\right)
$$

Finally, we can construct a pair wise comparison matrix according firstly to thirdly with all expert's opinion.

3) Applying $\tilde{Z}$ into FAHP.

\section{An example of application Z- FAHP}

Many factors lead to unpredictable and uncertain conditions in the process of tunneling. Underground construction will not only be accompanied by dangerous but also affect the engineering economy. If the decision-makers use improper methods will result in unimaginable losses. Therefore, it is a hot topic for scholar how to properly assess the level of risk in the mechanized tunneling. Hamidreza Nezarat et al. [13] by contribution of experienced technicians and use of geological study eight levels of risks are considered. They use FAHP to assess the level of risk and get good results. On the basis of their research, we add the concept of Z-number to improve the reliability of the evaluation.

According to Nezarat et al. [13]. we can make the hierarchical structure of geological risk problems in Figure 2.

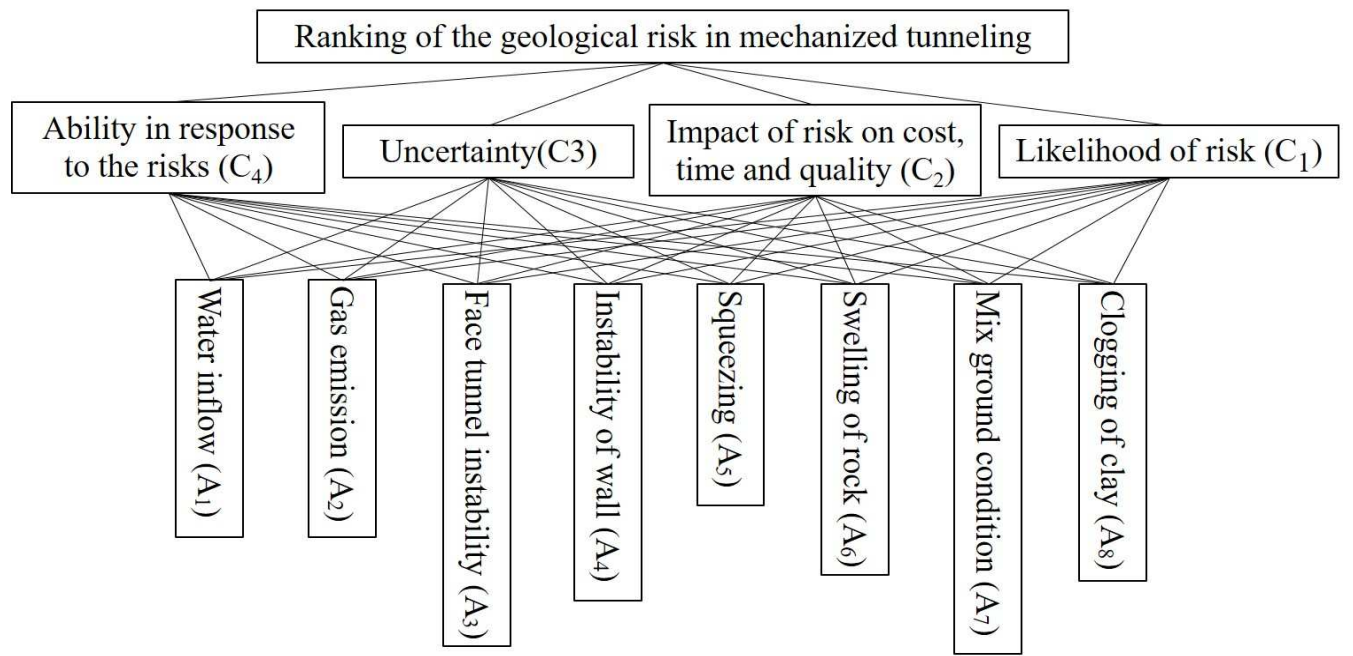

Figure 2: Hierarchical structure of the problem

Step 2: Each expert gives his or her opinion about decision variable with Z-number 
Sheng-sheng Zhang

Table 1: Z-number weight of decision variable

\begin{tabular}{c|c|c|c|c|c|c}
\hline$Z$ - number weight & \multicolumn{3}{|c|}{$A$} & \multicolumn{3}{|c}{$B$} \\
\cline { 2 - 7 } of decision variable & $a$ & $b$ & $c$ & $a$ & $b$ & $c$ \\
\hline$C_{1}$ & 1 & 1 & 1 & 0.8 & 0.9 & 1 \\
\hline$C_{2}$ & 1 & 3 & 5 & 0.75 & 0.875 & 1 \\
\hline$C_{3}$ & 1 & 2 & 4 & 0.8 & 0.9 & 1 \\
\hline$C_{4}$ & 1 & 1 & 3 & 0.75 & 0.875 & 1 \\
\hline
\end{tabular}

Step 3: Convert Z-number to a crisp number

a) Convert $B$ into crisp number according Eq6

Table 2: $\alpha$ of each decision variable value

\begin{tabular}{c|c|c|c|c}
\hline the value of $\alpha$ & $C_{1}$ & $C_{2}$ & $C_{3}$ & $C_{4}$ \\
\hline$\alpha$ & 0.9 & 0.875 & 0.9 & 0.875 \\
\hline
\end{tabular}

b) Convert weighted Z-number to regular fuzzy number according Eq9.

Table 3: Fuzzy weight of decision variable

\begin{tabular}{c|c|c|c}
\hline $\begin{array}{c}\text { Fuzzy weight of } \\
\text { decision variable }\end{array}$ & $a$ & $b$ & $c$ \\
\hline$C_{1}$ & 0.948683 & 0.948683 & 0.948683 \\
\hline$C_{2}$ & 0.935414 & 2.80624 & 4.67707 \\
\hline$C_{3}$ & 0.948683 & 1.89737 & 3.79473 \\
\hline$C_{4}$ & 0.935414 & 0.935414 & 2.80624 \\
\hline
\end{tabular}

Step 4: Construct the paired comparison matrix

According to the criteria of the paired comparison matrix in AHP, we can complete the paired comparison matrix.

Table 4: Fuzzy pair-wise comparison of decision variable

\begin{tabular}{c|c|c|c|c|c|c|c|c|c|c|c|c}
\hline & \multicolumn{4}{|c|}{$C_{1}$} & \multicolumn{3}{c|}{$C_{2}$} & \multicolumn{3}{c|}{$C_{3}$} & \multicolumn{3}{c}{$C_{4}$} \\
\hline & $a$ & $b$ & $c$ & $a$ & $b$ & $c$ & $a$ & $b$ & $c$ & $a$ & $b$ & $c$ \\
\hline$C_{1}$ & 1 & 1 & 1 & 0.20 & 0.34 & 1.01 & 0.25 & 0.5 & 1 & 0.34 & 1.01 & 1.01 \\
\hline$C_{2}$ & 0.94 & 2.81 & 4.68 & 1 & 1 & 1 & 0.94 & 1.87 & 3.74 & 1.87 & 3.74 & 5.61 \\
\hline$C_{3}$ & 0.95 & 1.90 & 3.79 & 0.25 & 0.5 & 1 & 1 & 1 & 1 & 0.95 & 2.85 & 4.74 \\
\hline$C_{4}$ & 0.94 & 0.94 & 2.81 & 0.15 & 0.24 & 0.47 & 0.21 & 0.35 & 1.05 & 1 & 1 & 1 \\
\hline
\end{tabular}

Step 5: Normal weight of decision variable 
A Model of Based on Z-number and Fuzzy Analytic Hierarchy Process

In the text, we use the extent FAHP [15]. In [15] Chang, D. Y. introduced the "Applications of The Extent Analysis Method on Fuzzy-AHP."

a) After the formation of the Pair-wise comparison matrix of weight vectors to be determined using fuzzy analytic hierarchy, the calculation of the not normal weights of decision variable using the method of Nezarat, Hamidreza, Farhang Sereshki, and Mohammad Ataei [13]. The results are shown in Table 5.

Table 5: Normal weight of decision variable

\begin{tabular}{c|c|c|c|c}
\hline \multicolumn{1}{c|}{ decision variable } & $C_{1}$ & $C_{2}$ & $C_{3}$ & $C_{4}$ \\
\hline $\begin{array}{l}\text { Not normal weight of } \\
\text { decision variable }\end{array}$ & 0.384 & 1 & 0.832 & 0.479 \\
\hline
\end{tabular}

b) Let the weight vectors normalize.

Table 6: Normal weight of decision variable

\begin{tabular}{c|c|c|c|c}
\hline \multicolumn{1}{c|}{ decision variable } & $C_{1}$ & $C_{2}$ & $C_{3}$ & $C_{4}$ \\
\hline $\begin{array}{l}\text { Not normal weight of } \\
\text { decision variable }\end{array}$ & 0.142 & 0.371 & 0.309 & 0.178 \\
\hline
\end{tabular}

Step 6: Normal weights of all criteria

We can calculate normal weights of all criteria with step 2 to step5 (see Table 7).

Table 7: Normal weights of all criteria

\begin{tabular}{c|c|c|c|c}
\hline Criteria & \multicolumn{4}{|c}{ Normal weight } \\
\hline$A_{1}$ & 0.124 & 0.145 & 0.144 & 0.122 \\
\hline$A_{2}$ & 0.108 & 0.105 & 0.13 & 0.158 \\
\hline$A_{3}$ & 0.145 & 0.163 & 0.109 & 0.113 \\
\hline$A_{4}$ & 0.136 & 0.138 & 0.137 & 0.084 \\
\hline$A_{5}$ & 0.153 & 0.181 & 0.11 & 0.128 \\
\hline$A_{6}$ & 0.121 & 0.09 & 0.115 & 0.133 \\
\hline$A_{7}$ & 0.119 & 0.088 & 0.113 & 0.131 \\
\hline$A_{8}$ & 0.094 & 0.091 & 0.142 & 0.134 \\
\hline
\end{tabular}

Step 7: Synthesize the solution for the ranking of geological risks in the problems of mechanized tunneling.

We have gotten the Normal weight of decision variable and normal weights of all criteria from step 1 to step 6 . Now we can find the global weight of all criteria in multiplying the global weight of each decision variable with the global weight of each criterion, respectively, and adding the resulting values in Table 8 . 


\section{Sheng-sheng Zhang}

Table 8: Coefficient matrix of pair-wise comparisons and rating of each criterion.

\begin{tabular}{|c|c|c|c|c|c|}
\hline \multirow{2}{*}{ Weight } & $C_{1}$ & $C_{2}$ & $C_{3}$ & $C_{4}$ & \multirow{2}{*}{ Sum } \\
\cline { 2 - 5 } & 0.142 & 0.371 & 0.309 & 0.178 & \\
\hline$A_{1}$ & 0.124 & 0.145 & 0.144 & 0.122 & 0.1376 \\
\hline$A_{2}$ & 0.108 & 0.105 & 0.13 & 0.158 & 0.1226 \\
\hline$A_{3}$ & 0.145 & 0.163 & 0.109 & 0.113 & 0.1349 \\
\hline$A_{4}$ & 0.136 & 0.138 & 0.137 & 0.084 & 0.1278 \\
\hline$A_{5}$ & 0.153 & 0.181 & 0.11 & 0.128 & 0.1457 \\
\hline$A_{6}$ & 0.121 & 0.09 & 0.115 & 0.133 & 0.1098 \\
\hline$A_{7}$ & 0.119 & 0.088 & 0.113 & 0.131 & 0.1098 \\
\hline$A_{8}$ & 0.094 & 0.091 & 0.142 & 0.134 & 0.1148 \\
\hline
\end{tabular}

From Table 8, we can see that the ranking of risks in Global tunnel with Z-FAHP in Global tunnel. Mix ground condition $\left(A_{7}\right)$ and Swelling of rock $\left(A_{6}\right)$ have the same risk level. Clogging of clay $\left(A_{8}\right)$ is higher than $A_{6}$. And Gas emission $\left(A_{2}\right)$ has higher rank than $A_{8}$. Squeezing $\left(A_{5}\right)$ has the highest risks. The second is Water inflow $\left(A_{1}\right)$. And the third is Face tunnel instability $\left(A_{3}\right)$. All in all, the rank of geological risks with ZFAHP is $\left(A_{5}>A_{1}>A_{3}>A_{4}>A_{2}>A_{8}>A_{6}=A_{7}\right)$. Clearly, we get the different result compared to Nezarat, Hamidreza, Farhang Sereshki, and Mohammad Ataei [13]. Therefore, we can use different methods according to different scenes and concerns. And we can also take into account Generalized Dodecagonal Fuzzy Numbers Using Incentre of Centroids [19] to rank risks.

\section{Conclusion}

This paper introduces the FAHP based on Z-number, which enhances the reliability of traditional FAHP. The general FAHP doesn't take into consideration the reliability of information effectively. Z-number is a new notion has more ability to describe the uncertain knowledge. In one way, two components of the Z-number are grouped together to convert it into a traditional fuzzy number. Then we use FAHP to solve the problems of MCDM. Through example we can see that the result of Z-FAHP is different from FAHP. The concept of Z-number is developing, which has still a long way to become mature. In the future, it will be applied to the problems of more decision making.

Acknowledgements. This work is supported by the Student Innovation and Entrepreneurship Project Training Program Chongqing University of Posts and Telecommunications University (No. 11).

\section{REFERENCES}

1. R.E.Bellman and L.A.Zadeh. Decision-making in a fuzzy environment, Management Science, 17 (1970) B-141-163. 
A Model of Based on Z-number and Fuzzy Analytic Hierarchy Process

2. C.Kahraman, ed. Fuzzy multi-criteria decision making: theory and applications with recent developments, Vol. 16. Springer Science \& Business Media, 2008.

3. G.Bordogna and P.Gabriella, A fuzzy linguistic approach generalizing boolean information retrieval: A model and its evaluation, Journal of the American Society for Information Science, 44 (1993) 70-79.

4. M.Dağdeviren and İ.Yüksel, Developing a fuzzy analytic hierarchy process (AHP) model for behavior-based safety management, Information Sciences, 178 (2008) 1717-1733.

5. F.Partovi, J.Burton and A.Banerjee, Application of analytical hierarchy process in operations management, International Journal of Operations \& Production Management, 10 (1990) 5-19.

6. J.K.Hamidi, et al., Risk assessment based selection of rock TBM for adverse geological conditions using Fuzzy-AHP, Bulletin of Engineering Geology and the Environment, 69 (2010) 523-532.

7. A.Mardani, A.Jusoh and E.K.Zavadskas, Fuzzy multiple criteria decision-making techniques and applications-Two decades review from 1994 to 2014, Expert Systems with Applications, 42 (2015) 4126-4148.

8. H.J.Zimmermann, Fuzzy Set Theory—and its Applications, Springer Science \& Business Media, 2011.

9. L.A.Zadeh, A note on Z-numbers, Information Sciences, 181 (2011) 2923-2932.

10. D.Dubois and H.Prade, The mean value of a fuzzy number, Fuzzy Sets and Systems, 24 (1987) 279-300.

11. B.Kang, et al., A method of converting Z-number to classical fuzzy number, Journal of Information and Computational Science, 9 (2012) 703-709.

12. Ch. Ch. Chou, The canonical representation of multiplication operation on triangular fuzzy numbers, Computers \& Mathematics with Applications, 45 (2003) 1601-2610.

13. H.Nezarat, F.Sereshki and M.Ataei, Ranking of geological risks in mechanized tunneling by using Fuzzy Analytical Hierarchy Process (FAHP), Tunnelling and Underground Space Technology, 50 (2015) 358-364.

14. L.A.Zadeh, A fuzzy-set-theoretic interpretation of linguistic hedges, Journal of Cybernetics, 2 (1972) 4-34.

15. D.Y.Chang, Applications of the extent analysis method on fuzzy AHP, European Journal of Operational Research, 95 (1996) 649-655.

16. L.A.Zadeh, Fuzzy sets, Information and Control, 8 (1965) 338-353.

17. L.Mikhailov and P.Tsvetinov, Evaluation of services using a fuzzy analytic hierarchy process, Applied Soft Computing, 5 (2004) 23-33.

18. A.Azadeh, et al., Z-AHP: A Z-number extension of fuzzy analytical hierarchy process, Digital Ecosystems and Technologies (DEST), 2013 7th IEEE International Conference on. IEEE, 2013.

19. J.P.Singh and N.I.Thakur, Ranking of generalized dodecagonal fuzzy numbers using incentre of centroids, Journal of Mathematics and Informatics, 5 (2016) 11-15. 